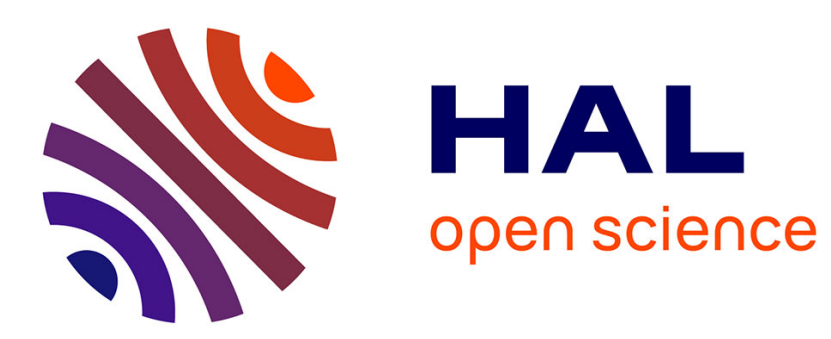

\title{
Direct computation of the noise generated by subsonic jets originating from a straight pipe nozzle
}

\author{
Christophe Bogey, Sébastien Barré, Christophe Bailly
}

\section{To cite this version:}

Christophe Bogey, Sébastien Barré, Christophe Bailly. Direct computation of the noise generated by subsonic jets originating from a straight pipe nozzle. International Journal of Aeroacoustics, 2008, 7 (1), pp.1-22. 10.1260/147547208784079917 . hal-00442510

\section{HAL Id: hal-00442510 https://hal.science/hal-00442510}

Submitted on 11 Apr 2016

HAL is a multi-disciplinary open access archive for the deposit and dissemination of scientific research documents, whether they are published or not. The documents may come from teaching and research institutions in France or abroad, or from public or private research centers.
L'archive ouverte pluridisciplinaire HAL, est destinée au dépôt et à la diffusion de documents scientifiques de niveau recherche, publiés ou non, émanant des établissements d'enseignement et de recherche français ou étrangers, des laboratoires publics ou privés. 


\title{
Direct computation of the noise generated by subsonic jets originating from a straight pipe nozzle
}

\author{
Christophe Bogey ${ }^{a, *}$, Sébastien Barré ${ }^{b}$ and Christophe Baillyc \\ Laboratoire de Mécanique des Fluides et d'Acoustique, \\ UMR CNRS 5509, Ecole Centrale de Lyon, 69134 Ecully Cedex, France
}

\begin{abstract}
The noise generated by isothermal round jets at Mach number $\mathrm{M}=0.9$ and at the diameter-based Reynolds number $\operatorname{Re}_{D}=5 \times 10^{5}$, originating from a straight pipe nozzle, is computed directly using Large Eddy Simulation (LES), in order to highlight the potential influence of the inlet boundary conditions on the acoustic predictions. Two jets are simulated, displaying levels of fluctuating axial velocity at the nozzle exit respectively of $1.6 \%$ and $9 \%$ of the jet velocity, while the momentum thickness of the shear layers is nearly the same. The shear-layer development and the radiated sound fields obtained for the two jets are found to differ significantly. The shear layer of the jet with low initial turbulence levels develops for instance with higher turbulence intensities and a velocity flow field that is more correlated. In addition coherent annular vortices and pairings are clearly observed in this jet. Regarding the radiated noise, the jet with high initial turbulence levels provides sound levels and spectra in fairly good agreement with experimental data obtained for jets at Reynolds numbers $\operatorname{Re}_{D} \geq 5 \times 10^{5}$. The jet with low nozzle-exit turbulence levels is shown to generate more noise, which might result from vortex pairings in the shear layer as it was suggested by Zaman [1].
\end{abstract}

\section{INTRODUCTION}

Computational aeroacoustics has made impressive advances over the last decade, and offers new perspectives on jet noise understanding and reduction, as it was pointed out by Tam [2] in 1998. The first challenge to overcome is however to show that the noise radiated by practical jets can be accurately predicted by numerical simulations, directly

\footnotetext{
*Christophe Bogey, Centre Acoustique, Ecole Centrale de Lyon, 36 avenue Guy de Collongue, 69134 Ecully Cedex, France Fax: 334721891 43, e-mail: christophe.bogey@ec-lyon.fr

${ }^{a}$ CNRS Research Scientist

${ }^{b}$ PhD., sebastien.barre@dassault-aviation.com, current address: Dassault Aviation, 78 quai Marcel Dassault, Cedex 300, 92552 Saint Cloud Cedex, France

${ }^{c}$ Professor at Ecole Centrale de Lyon and at Institut Universitaire de France, christophe.bailly@ec-lyon.fr
} 
by solving the unsteady compressible flow motion equations. With this aim in view, the development of Large Eddy Simulation (LES) techniques as well as the increase of computational power have led to significant progress, which are reported for instance in the recent review of Wang et al. [3]. Among the first LES of subsonic jet noise, the works of Zhao et al. [4], Bogey et al. [5, 6, 7], Bodony and Lele [8], and Rembold and Kleiser [9] can be particularly mentioned. They demonstrated the feasibility of the direct computation of jet noise using LES for cold and hot subsonic jets, both for round and rectangular geometries. Due to limitations in computer memory, these simulations could not however include the nozzle body in the computational domain, and the jet inflow was therefore specified by imposing meanflow profiles while adding lowamplitude disturbances to seed the shear-layer turbulence. This method results in uncertainties in the numerical predictions, because the jet development and the radiated noise obtained might appreciably depend on the characteristics of the forcing disturbances [10].

In order to improve LES of jet noise, it appears necessary to get rid of shear-layer forcing, and to include the nozzle body in the computational domain. Such computations have been performed by DeBonis and Scott [11], Biancherin et al. [12], Andersson et al. [13], Shur et al. [14], and Wu et al. [15], for cold and hot, subsonic and supersonic round jets. Note that DeBonis and Scott [11] provided only flow field results, whereas the other authors determined directly the radiated noise from their LES. In these works, no artificial forcing is applied in the shear layer near the nozzle exit. Nevertheless one might wonder whether the presence of the nozzle alone is sufficient to obtain accurate predictions of the jet flow and noise. The role of the nozzle in jet development and noise generation mechanisms is indeed still discussed. The presence of the nozzle is for instance known to be required for the investigation of the screech tones generated in supersonic jets, resulting from a feedback loop closing at the nozzle lip, as shown by the numerical achievements of Shen and Tam [16], Al-Qadi and Scott [17], Li and Guo [18], and Berland et al. [19]. However, for the mixing noise that is generated by the turbulence developing in the jet, the presence of the nozzle might not be enough to provide reliable results, because the properties of the boundary layer at the nozzle exit are likely to have significant effects on the jet flow, and therefore have to be considered.

In round jets, the characteristics of the boundary layer at the nozzle exit are actually observed to vary considerably. They depend in particular on the nozzle geometry [20], and on the diameter-based Reynolds number $\mathrm{Re}_{D}$. Zaman [21] for example noticed experimentally that jets can be expected to be initially laminar for $\operatorname{Re}_{D} \leq 10^{5}$, but initially turbulent for $\operatorname{Re}_{D} \geq 5 \times 10^{5}$. The resulting changes in the fluctuation levels at the nozzle exit are very important, and can affect the mean and turbulent development of the shear layer in a dramatic way according to Hussain and Zedan [22].

Husain and Hussain [23] further reported that the peaks of turbulence intensity in a developing shear layer are higher in an initially laminar jet than in an initially turbulent jet, which is expected to have an impact on the noise generated by the jets. Near-field measurements and LES fields $[10,24]$ indeed support that there are links between the peaks of turbulence in the shear layer and the sound sources. As it was suggested by Crighton [25] 
in the early eighties, the state of the nozzle-exit boundary layer may thus modify jet-noise mechanisms. The result can be for instance the presence of additional noise sources in initially laminar jets, as shown experimentally by Zaman [1], Bridges and Hussain [26], and Viswanathan [27]. Zaman [1] in particular demonstrated clearly that an initially laminar jet emits more noise than an initially turbulent jet, and attributes this additional noise to vortex pairings in the transitional shear layer. At this point, it is tempting to relate this additional noise to the overestimation of the sound pressure levels that is often obtained in LES of transitional jets at high Reynolds numbers, with or without nozzle [12, 28].

In the present work, isothermal round jets at Mach number $\mathrm{M}=\mathrm{u}_{j} / \mathrm{c}_{\mathrm{a}}=0.9$ and Reynolds number $\operatorname{Re}_{D}=\mathrm{u}_{\mathrm{j}} D / v=5 \times 10^{5}$ ( $\mathrm{u}_{j}$ is the jet inflow velocity, $D$ is the nozzle diameter, $\mathrm{c}_{\mathrm{a}}$ is the speed of sound in the ambient medium, $v$ is the kinematic molecular viscosity) are computed by compressible LES using low-dissipation and low-dispersion numerical schemes. The computational grid is cylindrical, and for the sake of simplicity includes a straight pipe nozzle, as in the recent LES of Uzun and Hussaini [29]. The turbulence intensities at the nozzle exit are specified notably by taking into account the flow inside the pipe. Two jets are thus considered, exhibiting root-mean-square peak levels of fluctuating axial velocity at the nozzle exit of $0.016 \mathrm{u}_{j}$ and $0.090 \mathrm{u}_{j}$, while the momentum thickness of the shear layers is nearly the same. To investigate the influence of the initial turbulence levels on the results, the flow fields obtained will be compared, with a particular attention given to the shear-layer zone. The near and far field sound pressure will be studied, and compared with experimental data provided in the literature for jets at high Reynolds numbers $\operatorname{Re}_{D} \geq 5 \times 10^{5}$. In this way, the agreement between the noise radiated by the jet with high turbulence levels at the nozzle exit and that of practical jets will be discussed. The presence of additional noise in the jet with low exit turbulence levels will also be tracked.

The paper is organized as follows. In section 2, the parameters of the numerical procedure and of the two simulated jets are defined. The calculation of the flow inside the pipe and the exit turbulence levels are also briefly shown. The flow and sound pressure fields obtained for the jets are compared in section 3: snapshots of vorticity and pressure are presented, properties of the mean flow and turbulent fields are reported, and the sound pressure near and far fields radiated by the jets are characterized in terms of levels, azimuthal cross-correlations and spectra. Concluding remarks are finally drawn in section 4 .

\section{SIMULATION PARAMETERS 2.1. Numerical procedure}

The turbulent flow and the radiated acoustic field of the subsonic jets are both computed directly by compressible LES. The cylindrical filtered Navier-Stokes equations are solved using numerical schemes providing low dispersion and low dissipation [30, 31]. The cylindrical coordinates are chosen in order to properly describe the jet geometry. The singularity on the axis is taken into account by the method proposed by Constantinescu and Lele [32], which is based on series expansions. The spatial discretization is performed by an eleven-point-stencil finite-difference scheme optimized in the wave-number space guaranteeing accuracy up to four points per wavelength. An optimized explicit six-stage Runge-Kutta algorithm is used for time integration. To ensure stability, grid-to-grid 


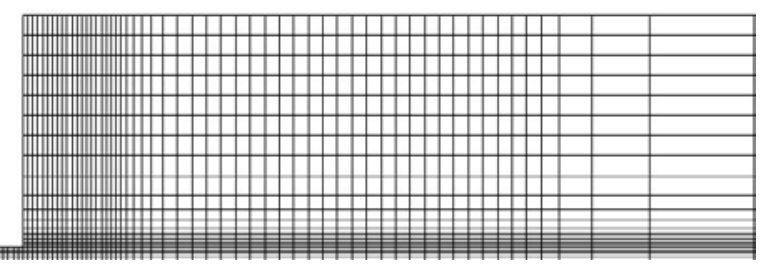

Figure 1. Visualization in the $(x, r)$ plane of the grid used for the jet simulations. Only every tenth line is shown (only $r \geq 0$ is plotted, the grid is symmetric).

oscillations are removed by applying explicitly to the flow variables an eleven-point-stencil selective filter. This filtering was designed so that only the short waves discretized by less than four points per wavelength are damped. Therefore it does not affect significantly the resolved scales, and takes account of the dissipative effects of the subgrid scales [33]. The present LES approach is indeed developed not to artificially decrease the effective Reynolds number of the jet, as it might be the case using dissipative subgrid modellings [34, 35]. Finally, in order to compute the noise directly, non-reflective boundary conditions are implemented, with the addition of a sponge zone at the outflow [28].

In the present jet simulations, the computational domain is made of two overlapping grids, as illustrated in figure 1 . The pipe grid has $n_{x} \times n_{r} \times n_{\phi}=391 \times 46 \times 48$ points, and is used to specify the nozzle-exit conditions. The main grid contains $n_{x} \times n_{r} \times n_{\phi}=$ $551 \times 219 \times 48$ points, and includes the lips of the pipe nozzle, the jet flow and a part of the acoustic field. In the radial direction, the grids are refined inside the boundary layer and the shear layer with a mesh spacing $\Delta r=0.009 \mathrm{D}$. The radial spacing then increases towards the jet centerline at the rate of $2 \%$ to reach $\Delta r=0.016 D$ on the axis, and outside the jet at the rate of $3 \%$ to obtain $\Delta r=0.066 D$ in the acoustic field. The grid extends radially up to $\Delta r=8.6 D$, and the sound waves are accurately calculated in the acoustic field up to the Strouhal number $\mathrm{St}=f D / \mathrm{u}_{\mathrm{j}} \simeq 3.3$ ( $f$ is the frequency). In the axial direction, the grid spacing is constant inside the pipe and up to $\Delta x=2.6 \mathrm{D}$ on the main grid, with the mesh spacing $\Delta x=0.016 D$. The grid is then stretched at the rate of $2 \%$ to obtain $\Delta x=0.047 D$. In addition the sponge zone begins at $x=16.4 D$. Considering the grid parameters, the length of the nozzle pipe is $6.7 D$, and that of the overlapping region between pipe and main grids is $0.61 D$. The azimuthal direction is discretized by the uniform spacing $\Delta \phi=2 \pi / 48$. Finally, to ensure Courant-Friedrichs-Lewy stability, the simulation time step is $\Delta t=1.1 \Delta r \Delta \phi / \mathrm{c}_{\mathrm{a}}$, where $\Delta r$ is the radial spacing on the axis. The simulation time is $1.02 \times 10^{5} \Delta t$, including a transitory period of $2.5 \times 10^{4} \Delta t$. The physical time $T$ therefore corresponds to $T u_{j} / D=160$.

\subsection{Jet specifications}

Two isothermal round jets $\left(T_{j}=T_{a}\right)$ with Mach and Reynolds numbers $\mathrm{M}=u_{j} / c_{a}=0.9$ and $\operatorname{Re}_{D}=u_{j} D / v=5 \times 10^{5}$ are simulated. The inflow velocity and the nozzle diameter of the two jets are thus $u_{j}=306 \mathrm{~m} \cdot \mathrm{s}^{-1}$ and $D=2.45 \mathrm{~cm}$, while the pressure and 
Table 1. Parameters of the two simulated jets: Mach and Reynolds numbers, and maximum RMS levels of the fluctuating axial velocity at the nozzle exit.

\begin{tabular}{lccc}
\hline & M & ReD & $\max \left(\mathbf{u}_{\mathbf{x}_{\text {rms }}^{\prime}}(\mathbf{x}=\mathbf{0})\right)$ \\
\hline jetH & 0.9 & $5 \times 10^{5}$ & $0.090 u_{j}$ \\
jetL & 0.9 & $5 \times 10^{5}$ & $0.016 u_{j}$
\end{tabular}

temperature of the ambient air are respectively $P_{a}=10^{5} \mathrm{~Pa}$ and $T_{a}=288 \mathrm{~K}$. In order to study the effects of the initial shear-layer turbulence level, the RMS peak level of fluctuating axial velocity at the nozzle exit is $0.090 u_{j}$ in the first jet and $0.016 u_{j}$ in the second jet, while the initial momentum thickness is about $\delta_{\theta}=0.008 D$ in the two cases. In what follows, these jets at high and low nozzle-exit turbulence levels will be respectively referred to as jetH and jetL, as shown in table 1 .

According to experiments of Zaman [21], for a round jet at Reynolds number $\operatorname{Re}_{D}=5 \times 10^{5}$, the boundary layer at the nozzle exit is expected to be turbulent, and its momentum thickness $\delta_{\theta}$ is of the order of $10^{-3} \mathrm{D}$. Such a boundary layer is too thin to be discretized with the computers that are now available. In the present simulations, a thicker boundary layer is consequently prescribed inside the pipe. More precisely, the momentum thickness at the nozzle exit is $\delta_{\theta}=0.0082 D$ for jetH and $\delta_{\theta}=0.0076 D$ for jetL.

In practice, a laminar boundary layer is arbitrarily imposed at the inflow of the nozzle pipe. It is defined by a polynomial approximation of a Blasius profile characterized by a thickness $\delta_{B}=0.064 D$ and

$$
\begin{aligned}
& u_{x}\left(r_{\mathrm{w}}\right)=u_{j} \frac{r_{\mathrm{w}}}{\delta_{B}}\left[2-2\left(\frac{r_{\mathrm{w}}}{\delta_{B}}\right)^{2}+\left(\frac{r_{\mathrm{w}}}{\delta_{B}}\right)^{3}\right] \text { if } r_{\mathrm{w}}<\delta_{B} \\
& u_{x}\left(r_{\mathrm{w}}\right)=u_{j} \text { if } r_{\mathrm{w}} \geq \delta_{B}
\end{aligned}
$$

where $r_{w}$ is the distance from the wall. At the inflow, temperature is determined by a Crocco-Busemann relation. In order to seed the turbulence, low-amplitude velocity fluctuations random in time and spatially uncorrelated are introduced in the boundary layer, at $x=-6.4 D$ far upstream inside the pipe. Unfortunately, due to computer limitations, it is not currently possible to describe accurately the spatial transition of the boundary layer. This led for instance Uzun \& Hussaini [29] to use a recycling procedure inside the pipe in their recent LES of round jet. In the present work, the boundary layer is under-resolved, and the radial, axial and azimuthal spacings at the wall are, in wall units, $\Delta r^{+}=36, \Delta x^{+}=63$, and $\left(\frac{1}{2} D \Delta \phi\right)^{+}=260$. Keep in mind that here the aim of the calculation inside the pipe is not to compute the boundary layer, as it can be done in Direct Numerical Simulations (DNS) [36], but to generate a nozzle-exit turbulence as realistic as possible for the LES of jet noise. In this way, there is no forcing applied directly in the jet flow [10]. The present method should also allow us to obtain high levels of turbulence at the jet inflow. Note finally that it has been recently used in the LES of a coaxial jet [37]. 
(a)

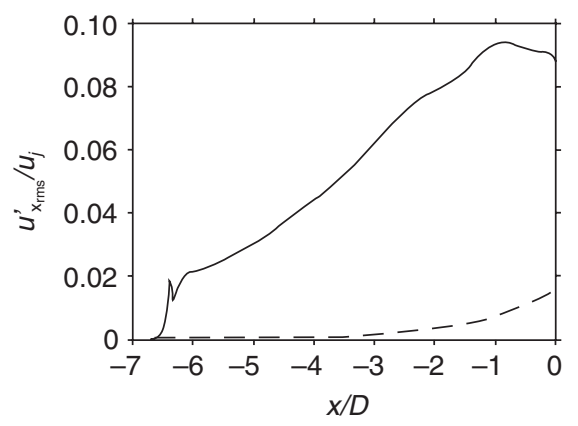

(b)

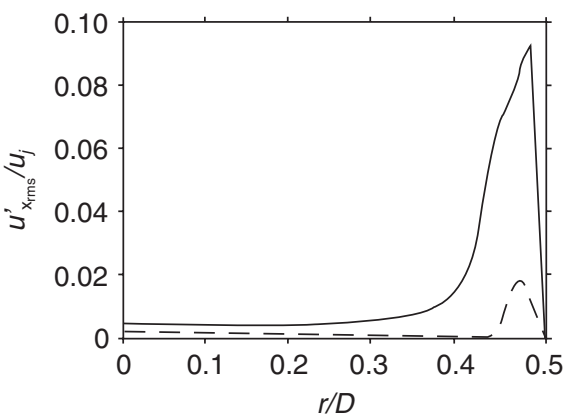

Figure 2. RMS levels of the fluctuating axial velocity. (a) Axial variations in the nozzle, along the line at $r=0.47 \mathrm{D}$ in the boundary layer, and (b) radial profiles at $x=0$ at the nozzle exit, obtained for: — - jetH; ---jetL.

\subsection{Nozzle-exit turbulence intensities}

In order to show how the nozzle-exit turbulence is specified in the two jets, the axial evolution of the levels of fluctuating axial velocity $u_{x_{\text {rme }}}^{\prime}$ at $r=0.47 D$, or $r^{+}=108$ in wall units, along the pipe, is presented in figure 2(a) for jetH and jetL. It can be first noticed the difference in amplitude of the random disturbances introduced at $x=-6.4 D$ in order to obtain the nozzle-exit turbulence levels reported in table 1. The RMS amplitude of these disturbances is indeed of $0.02 u_{j}$ for jetH, but only of the order of $0.001 u_{j}$ for jetL. These disturbances are growing along the pipe, to provide, at the nozzle exit, the radial profiles of $u_{x_{r m s}}^{\prime}$ presented in figure 2(b). As expected, the turbulence levels are important close to the wall, whereas $u_{x_{\text {rms }}}^{\prime} \simeq 0.005 u_{j}$ is found in the centerline region. The peak levels obtained correspond to the values specified in table 1 , that are $0.090 u_{j}$ for jetH and $0.016 u_{j}$ for jetL. It can be also noted that the axial evolution of the turbulence intensities in figure 2(a) suggests that in both jets the turbulent structures are still evolving as the pipe exit is approached. This is in particular the case in jetH with a drop of $u_{x_{m x}}^{\prime} / u_{j}$ near $x=-D$ indicating that turbulence is still transitional close to the nozzle exit.

The radial profiles of mean axial velocity obtained at the pipe exit for jetH and jetL are plotted in figure 3 . The profile for jetL is very similar to the initial Blasius profile at $x=-6.4 D$ inside the pipe, whereas that for jetH is slightly different. The growth of turbulent disturbances along the pipe boundary in jetH has thus led to a mean velocity profile which is sharper at the wall. Because turbulence is not fully developed at the pipe exit as mentioned previously, and because of the under-resolution of the boundary layer, this profile cannot however be compared with the profiles typically obtained for turbulent boundary layers [23]. Considering these issues, it will be interesting in future works to develop turbulent boundary layers at the pipe exit when computers with increasing power make it possible.

The RMS radial profiles of the fluctuating axial, radial and azimutal velocities at $x=$ $-0.6 D$ close to the nozzle exit are finally presented in figure 4 for jetH. Surprisingly, the relative shapes of the curves obtained for the different velocity components are in a fair 


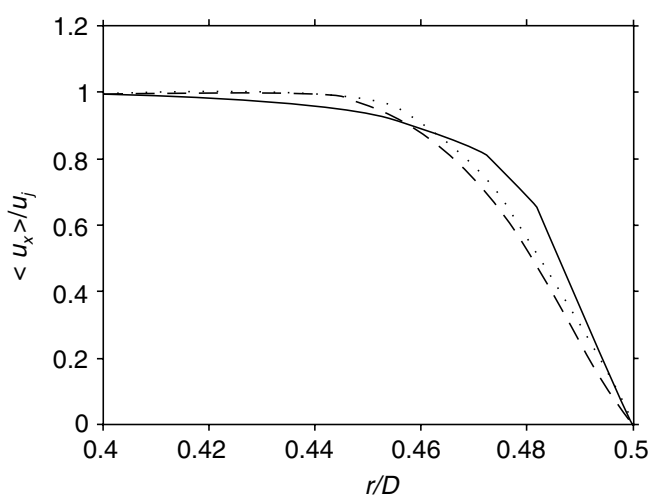

Figure 3. Radial profiles of mean axial velocity obtained at $x=0$ at the nozzle exit for — jetH and ---jetL; ....Blasius profile specified at $x=-6.4 D$ inside the pipe.

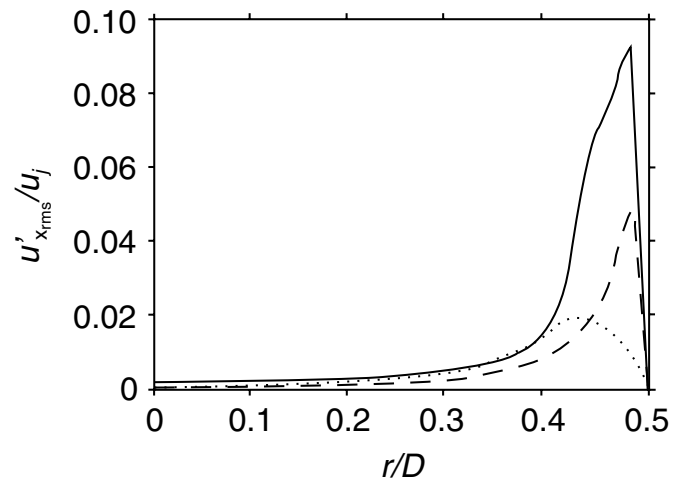

Figure 4. Radial profiles of the RMS levels of fluctuating velocities at $x=-0.6 D$ for jetH: — axial velocity $u_{x_{m s}}^{\prime} / u_{j}, \ldots$ radial velocity $u_{r_{m s}}^{\prime} / u_{j},---$ azimuthal velocity $u_{\phi_{r m s}}^{\prime} / u_{j}$.

agreement with the experimental and DNS data reported by Eggels et al. [36] for a turbulent pipe flow at a low Reynolds number. This agreement is only qualitative, the peak levels in jetH being for instance observed much farther from the wall, because of the underresolution in the present LES. High levels of turbulence at the nozzle exit are however obtained in jetH, in order to study the influence of jet inlet conditions on noise prediction.

\section{RESULTS}

\subsection{Snapshots of vorticity and pressure}

Snapshots of the vorticity fields obtained for jetH and jetL in the shear layer developing after the nozzle are shown in figure 5. For the jet with high initial levels of turbulence, in figure 5(a), small vortical structures are found very close to the nozzle exit, displaying rapidly typical features of three-dimensional mixing. For the jet with low nozzle-exit 
(a)

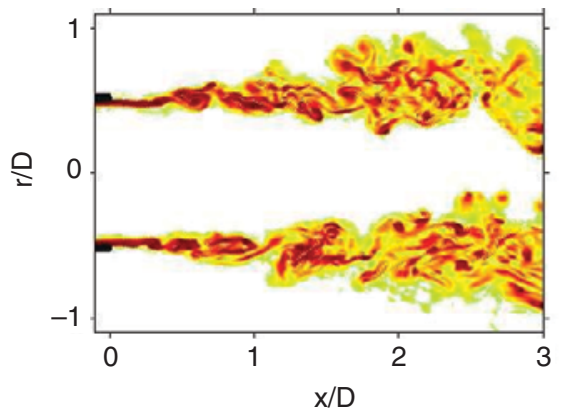

(b)

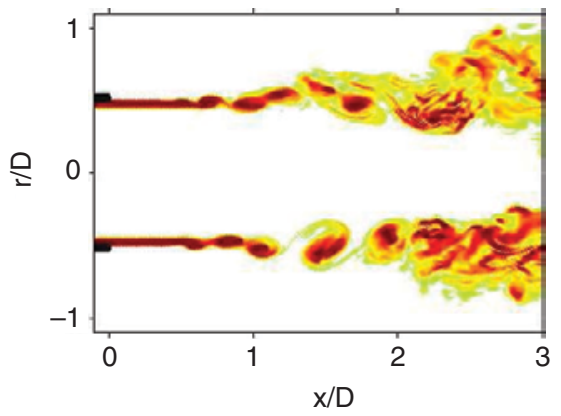

Figure 5. Snapshots in the $(x, r)$ plane of the vorticity norm $|\omega|$ obtained just downstream of the nozzle exit for: (a) jetH, (b) jetL. The color scale ranges up to the vorticity level of $2 \times 10^{5} \mathrm{~s}^{-1}$.

turbulence levels, in figure 5(b), the shear layer develops differently. It appears indeed laminar up to the location $\mathrm{x} \simeq 0.5 D$, where coherent vortices are generated. These vortices are convected in the downstream direction, and pairings occur clearly around $x=1.5 D$. Since vortex pairings are known as efficient noise generation mechanisms, there may be an impact on the radiated noise. After the pairings, the shear-layer turbulence then tends to be three-dimensional for $x \geq 2 D$.

In order to illustrate the noise radiated by the two jets, snapshots of the vorticity and of the sound pressure fields obtained on the whole computational domain are presented in figure 6. It might be hazardous to state from snapshots, but some comments can however be made especially in the light of the results presented later in section 3.3. The modelling of the initial fluctuations in the present compressible LES first does not seem to generate an overwhelming spurious noise at the pipe exit. The acoustic fields also suggest that the sound pressure levels emitted by jetL are higher than those of jetH. In particular strong acoustic waves propagating in the sideline direction are observed for jetL, see for instance the pressure field around $x=4 D$ and $r=4 D$ in figure 6(b), whereas the presence of this kind of waves is less clear for jetH. An additional radiation may therefore be found for the jet with low initial turbulence levels, and it seems from figure 6(b) to originate from the flow region at $x \simeq 2 D$ where the first stage of pairing of the coherent shear-layer vortices takes place. This result agrees with the experimental works of Zaman [1], and Bridges and Hussain [26] for initially laminar and turbulent jets.

\subsection{Mean and turbulent flow fields}

The mean and turbulent flow fields of the jets are now investigated. The mean axial velocity field and streamlines around the jet are first represented in figure 7 for jetH. Very similar results are obtained for jetL. The streamline pattern is in good agreement with experimental findings, and indicates that the entrainment of the fluid surrounding the jet into the flow occurs in an appropriate manner, and is not obstructed by the boundary conditions. 


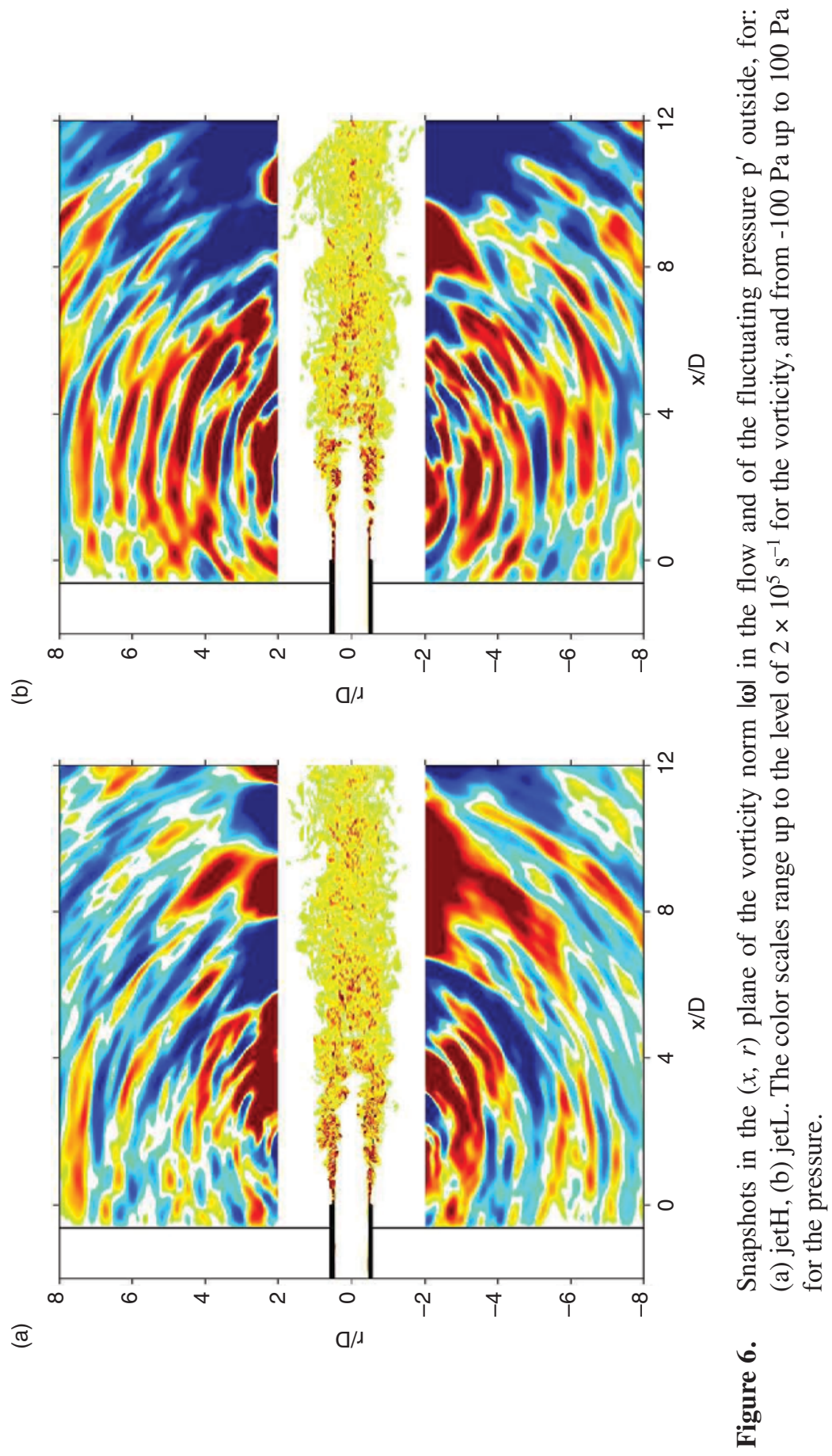




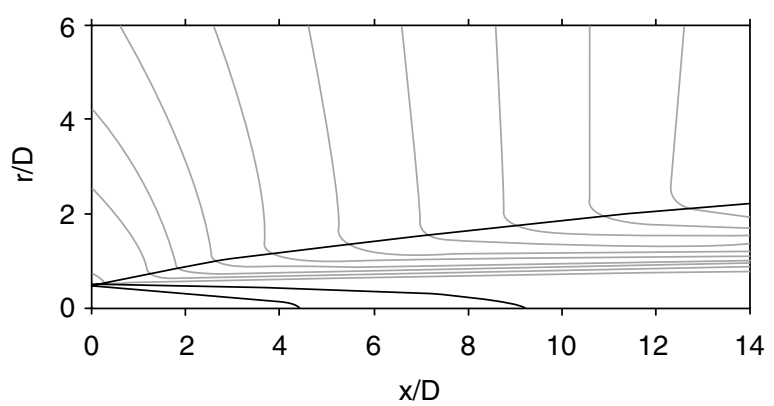

Figure 7. Mean flow obtained in the $(x, r)$ plane for jetH: streamlines (grey curves), and iso-contours of mean axial velocity corresponding to $0.95 u_{j}, 0.67 u_{j}$ and $0.025 u_{j}$ (black curves). Only $r \geq 0$ is plotted, the mean flow is symmetric.

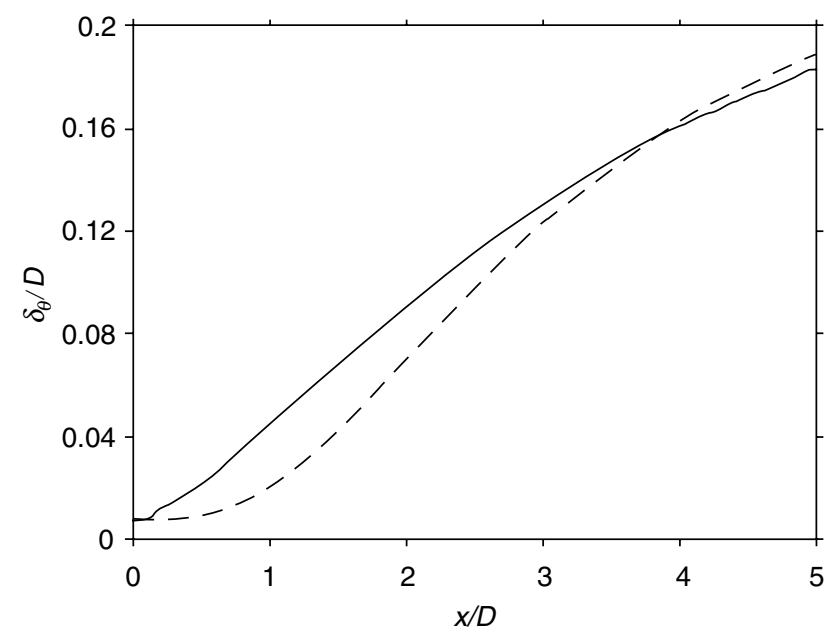

Figure 8. Axial evolution of the momentum thickness of the shear layer, obtained for:-_ jetH; ---jetL.

The length of the potential core $x_{c}$, defined here arbitrarily by $u_{c}\left(x_{c}\right)=0.95 u_{j}$ where $u_{c}$ is the jet mean centerline velocity, is $x_{c}=4.7 \mathrm{D}$ for both jets. This result suggests that the development of the shear layer, which occurs farther downtream in jetL than in jetH as shown in figure 5, is more rapid in jetL. This difference of behaviour is also illustrated in figure 8 by the evolution of the momentum thickness of the shear layer in the two jets. The shear-layer spreading is delayed in jetL with respect to jetH, but is then faster especially around $x=2 D$, in the region of vortex pairings. In JetH, the shear layer appears moreover to develop just downstream of the pipe exit, but two stages of linear growth cannot be observed as typically found in initially turbulent shear layers [23]. Therefore both jetL and jetH display features of initially transitional jets.

The present core length of $x_{c}=4.7 D$ is smaller than experimental results for Mach 0.9 jets $[38,39]$ at high Reynolds numbers $\operatorname{Re}_{D} \geq 5.0 \times 10^{5}$, which exhibit core 


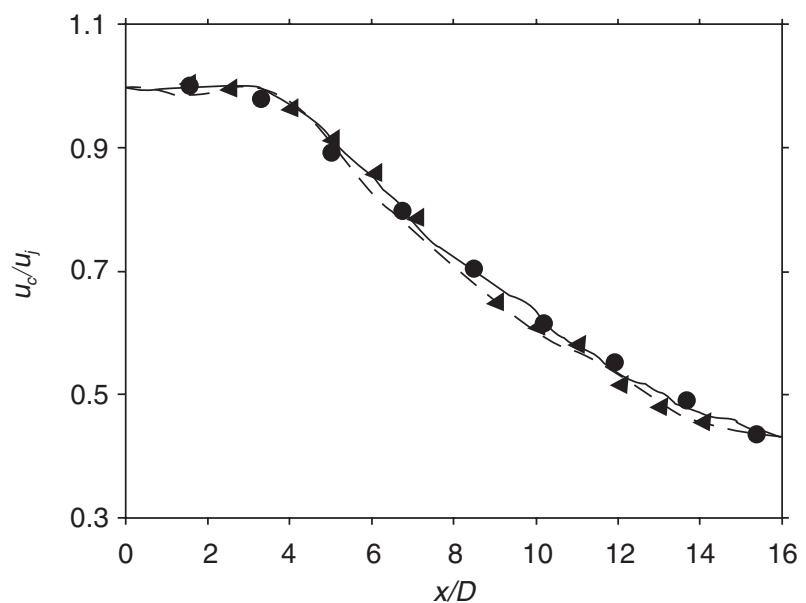

Figure 9. Profiles of centerline mean axial velocity, obtained for: — jetH; ---jetL. Measurements: 4 Lau et al. [38] $\left(\mathrm{M}=0.9, \mathrm{Re}_{D}=10^{6}\right)$, Arakeri et al. [39] $\left(\mathrm{M}=0.9, \mathrm{Re}_{D}=5 \times 10^{5}\right)$. For the comparison the experimental profiles are shifted in the axial direction with respect to the LES profiles to yield identical core lengths.

lengths of $x_{c} \simeq 7 D$. One can first mention the difference in momentum thickness at the nozzle exit, since $\delta_{\theta}=0.008 D$ is specified in the present jets whereas $\delta_{\theta} \simeq 0.001 D$ is expected in experimental jets at such Reynolds numbers [21]. The present core length must also be due to the fact that jets with initially transitional shear layer, including jetH and jetL, develop with shorter core length than initially turbulent jets, as clearly shown by Raman et al. [40] for tripped/untripped jets.

The variations of the mean axial velocity $u_{c}$ along the jet centerline are presented in figure 9 . The velocity decays obtained for jetH and jetL do not significantly differ, and agree with the velocity decays measured by Lau et al. [38] and by Arakeri et al. [39] for $\mathrm{M}=0.9$ jets at $\operatorname{Re}_{D}=10^{6}$ and $\operatorname{Re}_{D}=5 \times 10^{5}$, both experimental profiles being axially shifted for the comparison. Jet velocity decay was found in a previous work [33] to depend appreciably on the Reynolds number of the flow. The present LES therefore provide results in agreement with the high Reynolds number considered. Note also the small decrease in velocity near $x=2 D$ in jetL. A similar decrease was previously described for an experimental jet [41] at the moderate Reynolds number $\operatorname{Re}_{D}=5.3 \times$ $10^{4}$, and for a jet simulated by LES without nozzle [28].

The RMS levels of the fluctuating axial velocity $\mathrm{u}_{x_{\mathrm{rms}}}^{\prime}$ on the jet centerline are presented in figure 10. They rapidly increase at the end of the potential core, and reach a peak at about $x=x_{c}+D$ in jetL, and $x=x_{c}+2 D$ in jetH. The peak level obtained for jetL is higher than that for jetH $\left(0.14 \mathrm{u}_{\mathrm{j}}\right.$ versus $\left.0.12 u_{j}\right)$. Both peak values are in the range of experimental data for Mach number 0.9 jets $[38,39]$ at $\operatorname{Re}_{D} \geq 5.0 \times 10^{5}$. The very good agreement between the LES profile for jetH and the profile obtained recently by Arakeri et al. [39] using PIV measurements can also be noticed. 


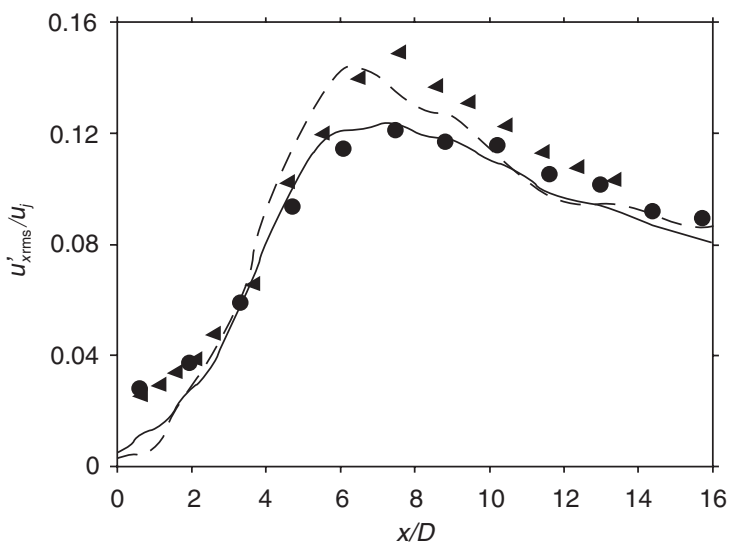

Figure 10. Centerline profiles of the RMS levels of the fluctuating axial velocity, obtained for: __ jetH; ---jetL. Measurements: 4 Lau et al. [38] $(\mathrm{M}=0.9$, $\left.\operatorname{Re}_{D}=10^{6}\right)$, A Arakeri et al. [39] $\left(\mathrm{M}=0.9, \operatorname{Re}_{D}=5 \times 10^{5}\right)$. For the comparison the experimental profiles are shifted in the axial direction with respect to the LES profiles.

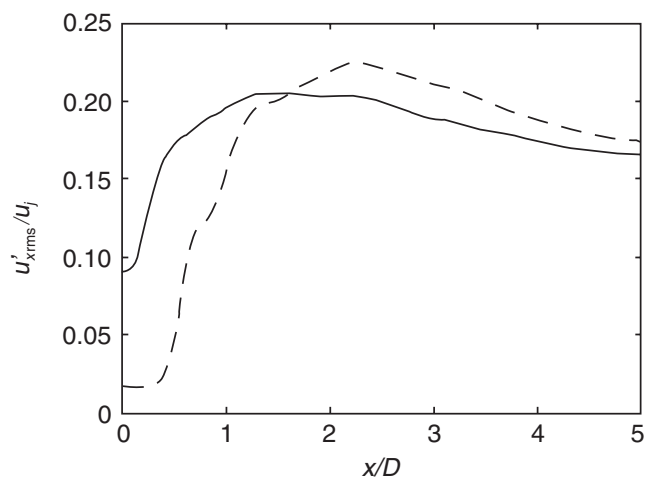

Figure 11. Profiles along the line at $r=0.48 D$ in the shear layer, of the RMS levels of the fluctuating axial velocity, obtained for: _ _ jetH; --- jetL.

We now focus on the shear-layer region. The RMS profiles of the fluctuating axial velocity levels along $r=0.48 D$ are plotted in figure 11 . Downstream from the nozzle exit, the values of $u_{x_{\text {rms }}}^{\prime}$ in the shear layer increase very rapidly, reach a peak and then slowly decrease. The level peak is closer to the nozzle, and has a lower amplitude in jetH than in jetL.

This behaviour is in agreement with the experimental results of Husain and Hussain [23], who showed that the turbulence levels in the early shear-layer development are lower in initially turbulent jets than in initially laminar jets. In initially laminar jets, they reported peaks of $u_{x_{m x}}^{\prime}$ at the axial locations $x \simeq 0.4 D$, with levels of 
(a)

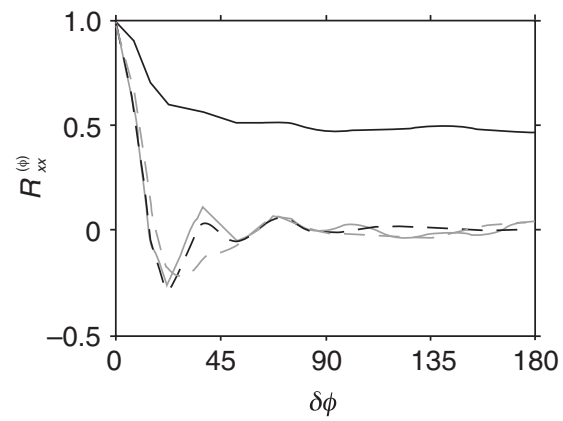

(b)

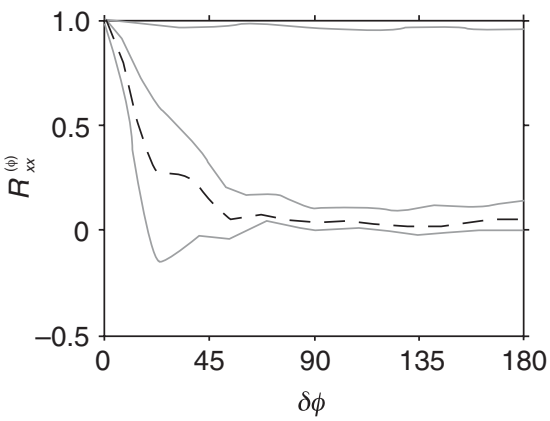

Figure 12. Azimuthal cross-correlation functions of the fluctuating axial velocity, at $r=D / 2$ in the shear layer for the axial locations: $-x=0$,

$-x=D / 2,--x=D,--x=2 D$, obtained for the jets: (a) jetH, (b) jetL.

about $0.18 u_{j}$. The development of the shear-layer turbulence in the present LES occurs farther downstream with a stronger intensity. This discrepancy with respect to experiments may result from the relatively thick initial momentum thickness of the shear layer specified in the simulations, which can appreciably affect the growth rates of instabilities [42]. Note also that in initially turbulent shear layers, $u_{x_{\operatorname{mov}}}$ does not exhibit any peak value but increases monotonically along the shear layer [23], which is not found here for the transitional jetH with high initial levels of turbulence intensity at the pipe exit.

In order to investigate some spatial properties of the shear-layer turbulence, azimuthal cross-correlations of the fluctuating axial velocity are calculated. The normalized correlation functions are defined by:

$$
R_{x x}^{(\phi)}(x, r, \delta \phi)=\frac{\left\langle u_{x}^{\prime}(x, r, \phi) u_{x}^{\prime}(x, r, \phi+\delta \phi)\right\rangle}{\left\langle u_{x}^{\prime}(x, r, \phi)\right\rangle^{1 / 2}\left\langle u_{x}^{\prime}(x, r, \phi+\delta \phi)\right\rangle^{1 / 2}}
$$

where $\delta \phi$ is the azimuthal separation angle, and $<\bullet>$ denotes statistical averaging. They are computed for $r=D / 2$ in the shear layer, at the axial locations $x=0, x=D / 2, x=D$ and $x=2 D$. They are represented in figure 12(a) for jetH and in figure 12(b) for jetL. For jetH, in the case with high initial turbulence intensities, the correlation levels at $x=$ 0 at the nozzle exit are close to 0.5 over the range $0 \leq \delta \phi \leq 180^{\circ}$. Since the random velocity disturbances introduced inside the pipe are non correlated in the azimuthal direction, this property is certainly due to the calculation of the boundary layer. The correlations however decrease rapidly downstream of the nozzle. They are in particular negligible for $\delta \phi \geq 90^{\circ}$ at $x=D / 2, x=D$ and $x=2 D$. The correlation levels are higher in jetL. At the nozzle exit, $R_{x x}^{(\phi)} \simeq 1$ is especially found for all azimuthal separation angles $\delta \phi$. Downstream, the correlation functions progressively exhibit lower values, to show, at $x=2 D$, levels roughly similar to those in jetH. In jetH the shear-layer velocity disturbances are therefore weakly correlated azimuthally just after the nozzle exit, whereas in jetL the velocity fluctuations in this region are more correlated. This feature 


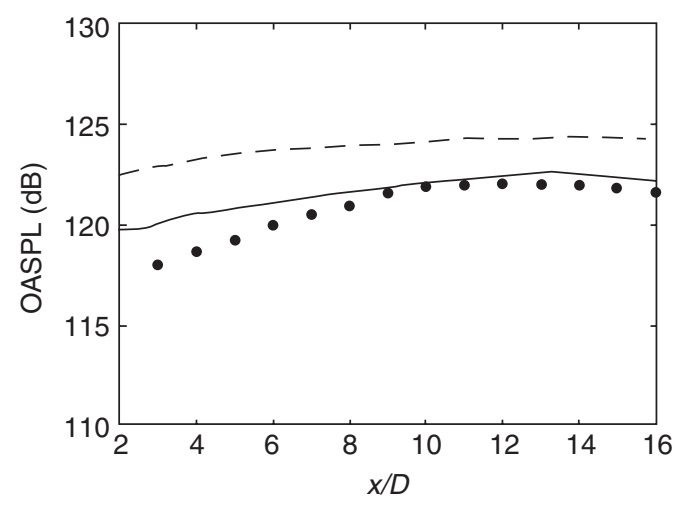

Figure 13. Overall sound pressure levels obtained along the line at $r=7.5 \mathrm{D}$, for the jets: — _ jetH, ---jetL. Measurements: $\bullet$ Bogey et al. [43] $\left(\mathrm{M}=0.9, \operatorname{Re}_{D}=\right.$ $\left.7.8 \times 10^{5}\right)$.

is also indicated by the presence of shear-layer coherent structures in the vorticity field of figure 5. The correlations in jetL become similar to those obtained in jetH only at $x=2 D$, after the first vortex pairings.

\subsection{Sound pressure field}

\subsubsection{Near acoustic field}

To investigate the noise radiated by the jets, properties of the near acoustic fields calculated directly by LES are first presented. The sound pressure levels along the line $r=$ $7.5 D$ are shown in figure 13. They are compared to the experimental data provided by Bogey et al. [43] for a jet at $\mathrm{M}=0.9$ and at the high Reynolds number $\operatorname{Re}_{D}=7.8 \times 10^{5}$. A fairly good agreement is observed between the sound levels obtained for the jet with significant initial turbulence levels and the measurements. The levels from jetH and from the experiment indeed do not differ by more than $2 \mathrm{~dB}$ at all the axial locations considered. With respect to jetH, jetL with low initial turbulence levels is found to generate sound levels higher by $4-5 \mathrm{~dB}$ for all emission angles. This behaviour is consistent with the pressure snapshots of figure 6 , and corresponds to the experimental results reported by Zaman [1], and Bridges and Hussain [26] for tripped and untripped subsonic jets. A similar overestimation was also found for the sound levels generated in the sideline direction by a high-Reynolds-number, initially laminar jet simulated without nozzle [28]. The present results illustrate the strong impact of nozzle-exit turbulence on the radiated noise. They also characterize quantitatively the additional noise observed in jetL.

To display the spatial structure of the acoustic fields of jetH and jetL, the azimuthal correlation functions of the fluctuating pressure obtained for $r=7.5 \mathrm{D}$ from the centerline, at the axial locations $x=0$ and $x=15 D$, are plotted in figure 14 . These functions are calculated in the following way:

$$
R_{x x}^{(\phi)}(x, r, \delta \phi)=\frac{\left\langle p^{\prime}(x, r, \phi) p^{\prime}(x, r, \phi+\delta \phi)\right\rangle}{\left\langle p^{\prime 2}(x, r, \phi)\right\rangle^{1 / 2}\left\langle p^{\prime 2}(x, r, \phi+\delta \phi)\right\rangle^{12}}
$$




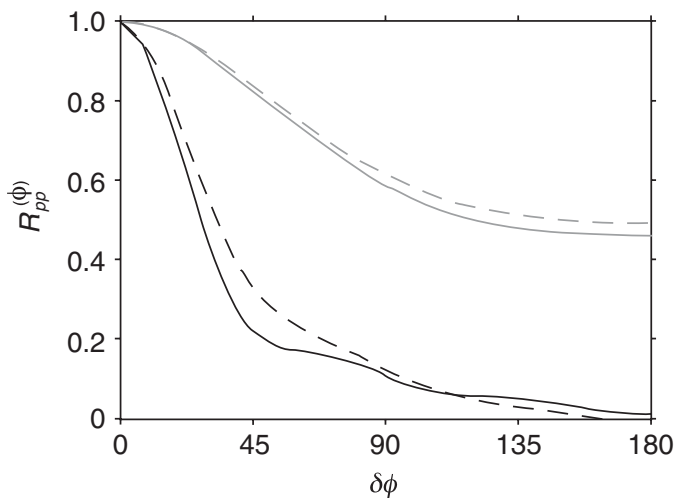

Figure 14. Azimuthal cross-correlation functions of the fluctuating pressure radiated by the jets: — jetH, --- jetL, at $r=7.5 D$ for the axial locations: $x=0$ (black curves) and $x=15 D$ (grey curves).

In agreement with experimental data [44] and numerical results [6], the correlation functions are seen to vary remarkably with the angle of radiation with respect to the jet direction. In both jetH and jetL, the correlation levels calculated at $x=15 D$ are indeed high for $0 \leq \delta \phi \leq 180^{\circ}$, whereas those obtained at $x=0$ decrease rapidly with the azimuth (note that the radiation angle, taken from the nozzle exit, is $\theta=27^{\circ}$ in the first case, but $\theta=90^{\circ}$ in the second case). Moreover the correlation levels are higher in jetL than in jetH. The azimuthal cross-correlations of the radiated pressure therefore display similarities with the correlations of figure 12 involving the velocity disturbances in the shear layer before the first vortex pairings.

\subsubsection{Far acoustic field}

The investigation of the noise radiated by turbulent flows is usually conducted in the far acoustic field. They are in particular generally based on far-field sound pressure spectra. The near acoustic fields calculated directly by LES for jetH and jetL are therefore now propagated much farther, up to about fifty diameters from the jet centerline. Practically, the flow quantities including density, velocity and pressure, provided by LES on two cylindrical surfaces at $r / D=4$ and $r / D=6$ surrounding the turbulent jets, are stored for $-0.5 D \leq x \leq$ $16 D$. They are then introduced in a propagation solver based on a simplified set of the flow motion equations, as done for instance by Gloerfelt et al. [45] for the computation of cavity noise. In the present work, the propagation is carried out by solving the Euler equations using the same numerical schemes and boundary conditions as the LES solver in order to obtain the same accuracy. The propagation grid mesh is uniform and contains $n_{x} \times n_{r} \times n_{\phi}$ $=821 \times 529 \times 48$ points, and enables to use a time step 12 times larger than the LES time step. The connection between LES and the Euler equations is illustrated in figure 15 with a snapshot of the pressure field obtained using the storage surface at $r / D=6$. The sound waves appear to be propagated without discontinuity at the joining surface. The results presented in what follows are calculated using the surface at $r / D=4$. 


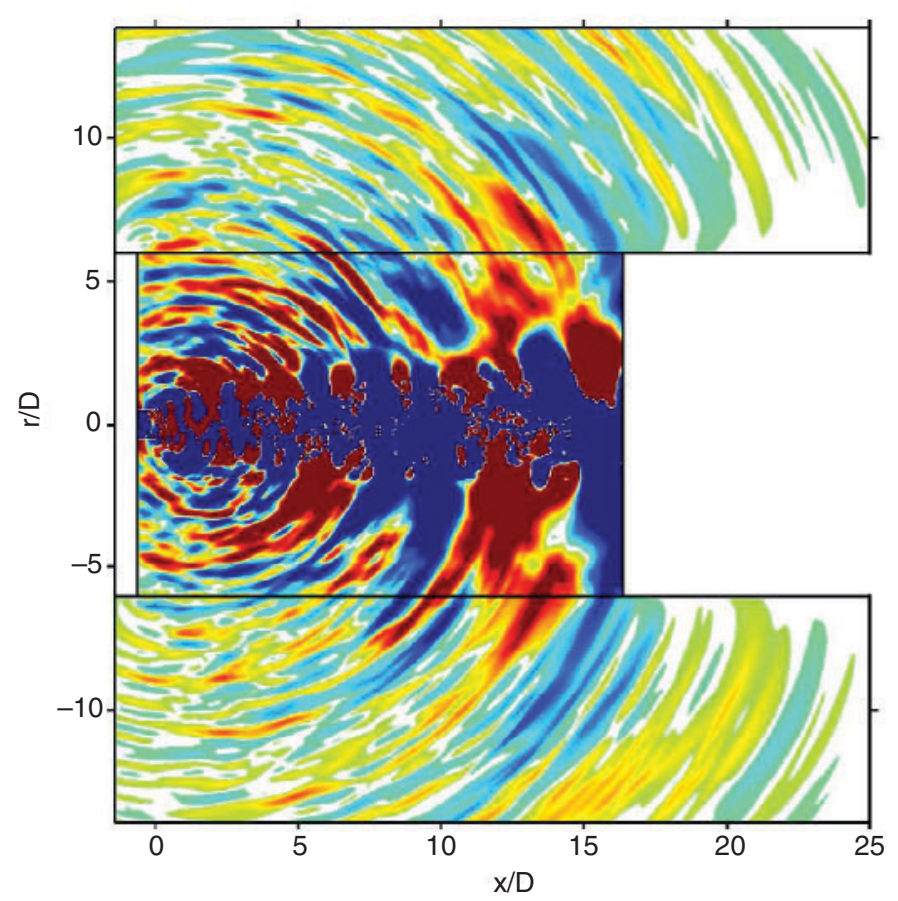

Figure 15. Snapshot in the $(x, r)$ plane of the fluctuating pressure $\mathrm{p}^{\prime}$ obtained for jetH. In the central region: pressure field provided by the LES computation, in the peripheral regions: pressure field given by the propagation solver based on the Euler equations.

The sound pressure levels calculated along the line located at $r=20 D$ are represented in figure 16. They display the same features as the results obtained at $r=7.5 \mathrm{D}$ previously shown in figure 13 . The acoustic levels predicted from jetH compare indeed favourably with the experimental data of Bogey et al. [43] for a jet at $\mathrm{M}=0.9$ and $\operatorname{Re}_{D}$ $=7.8 \times 10^{5}$, whereas the levels from jetL are overestimated by $4-5 \mathrm{~dB}$.

The narrow-band pressure spectra obtained at a distance of 52D from the nozzle exit for angles of $\theta=40^{\circ}$ and $\theta=90^{\circ}$ with respect to the jet direction are plotted in figures 17(a) and 17(b), respectively. At $\theta=40^{\circ}$, the spectrum computed for jetH is in good agreement with corresponding spectra measured by Bogey et al. [43] and by Jordan et al. [46] for jets with Reynolds numbers $\operatorname{Re}_{D} \geq 7.8 \times 10^{5}$. The maximum difference between levels of numerical and experimental spectra is indeed only of the order of 2 $\mathrm{dB}$ at low Strouhal numbers. However the peak Strouhal number in jetH is $\mathrm{St}_{p}=0.33$, which is higher than the $\mathrm{St}_{p} \simeq 0.20$ observed in experiments. The peak Strouhal number is therefore over-estimated, in the same way as in previous LES including no nozzle [10]. As for the spectrum from jetL, it displays the same shape as the spectrum from jetH, but exhibits levels higher by $1-2 \mathrm{~dB}$. 


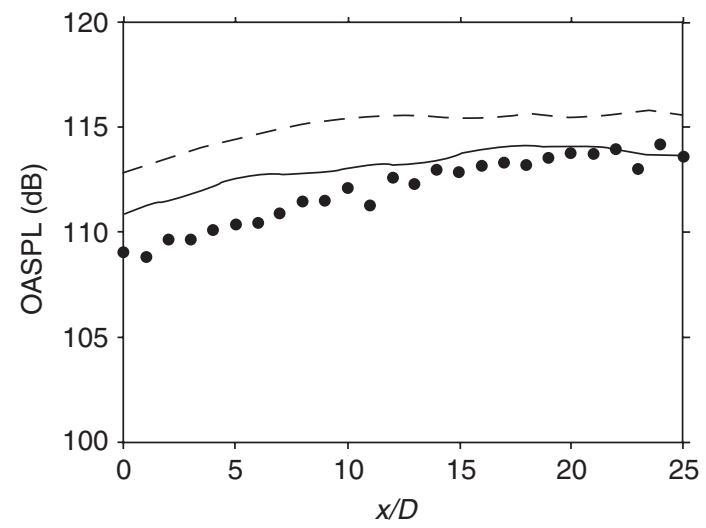

Figure 16. Overall sound pressure levels obtained along the line at $r=20 D$, for the jets: - jetH, --- jetL. Measurements: $\bullet$ Bogey et al. [43] $\left(\mathrm{M}=0.9, \operatorname{Re}_{D}=\right.$ $\left.7.8 \times 10^{5}\right)$.

(a)

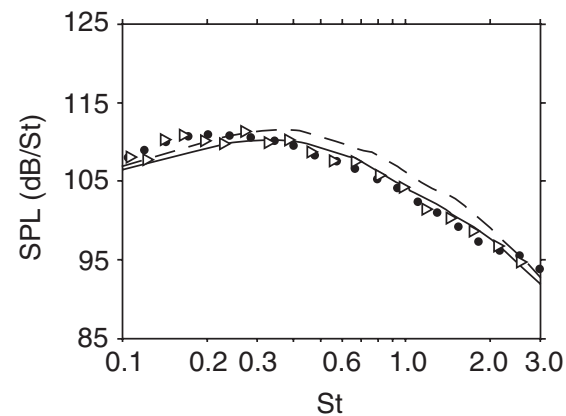

(b)

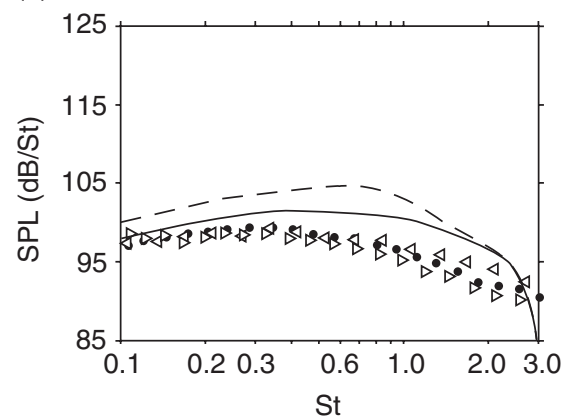

Figure 17. Narrow-band sound pressure spectra obtained at 52D from the nozzle exit for radiation angles: (a) $\theta=40^{\circ}$, (b) $\theta=90^{\circ}$, for the jets: — jetH, --- jetL, as function of Strouhal number $\mathrm{St}=\mathrm{f} D / u_{j}$. Measurements: $\bullet$ Bogey et al. [43] $\left(\operatorname{Re}_{D}=7.8 \times 10^{5}\right)$, Jordan et al. [46] $\left(\operatorname{Re}_{D}=10^{6}\right),<$ Tanna [47] $\left(\operatorname{Re}_{D}=10^{6}\right)$.

At $\theta=90^{\circ}$, the discrepancies between the spectra from jetH and jetL are more significant. With respect to jetH, the spectrum from jetL increases in amplitude, and is characterized by higher-frequency components with a peak Strouhal number $\mathrm{St}_{p}=0.7$. Zaman [1] and Bridges and Hussain [26] in their experiments conducted on tripped/untripped jets observed similar high-frequency peaks in the sideline pressure spectra for untripped jets. In terms of Strouhal number based on the initial momentum thickness of the shear layer $\mathrm{St}_{\theta}=\mathrm{f}_{\theta} / u_{j}$, these peaks were between 0.005 and 0.007 . In jetL, the frequency peak corresponds to a value of $\mathrm{St}_{\theta}=\mathrm{f \delta}_{\theta} / u_{j}=0.0053$, which is in the experimental range. Moreover, with respect to experimental data [43, 46, 47], the spectrum from jetH displays overestimated levels, especially at high Strouhal numbers. 
The Strouhal number peak is thus $\mathrm{St}_{p}=0.4$ in jetH, instead of $\mathrm{St}_{p}=0.3$ in experiments. The origin of this discrepancy is to be investigated, but it may be associated with the properties of the nozzle-exit boundary layer. In jetH, the initial momentum thickness is for instance larger than in experiments. The initial jet turbulence should be better resolved in future simulations.

\section{CONCLUSION}

The present Large Eddy Simulations of round jets at Mach number $\mathbf{M}=0.9$ and Reynolds number $\operatorname{Re}_{D}=5 \times 10^{5}$, originating from a straight pipe nozzle, enable to emphasize the effects of the levels of nozzle-exit turbulence. The effects are specially found to be significant on the shear layer development and on the sideline sound field. For high nozzle-exit turbulence levels, the sound field compares fairly well with that obtained for jets at high Reynolds numbers $\operatorname{Re}_{D} \geq 5 \times 10^{5}$. However, for low turbulence levels at the nozzle exit, coherent vortical structures are seen to develop and merge in the shear layer, and an additional noise increasing the sound levels by $4-5 \mathrm{~dB}$ is observed. For the computation of practical jets, the presence of the nozzle alone thus does not appear sufficient, and realistic initial conditions may be necessary to obtain relevant results. To meet this requirement, the difficulty is to generate turbulent conditions at the jet nozzle exit at an affordable computational cost, without producing spurious sound waves. One method is to calculate the boundary layer inside the nozzle, which however implies very fine discretization at the wall boundaries. Moreover, in order to improve the accuracy of noise predictions, there is a need for better taking into account the initial characteristics of practical jets. In particular, boundary layers with smaller momentum thickness and the nozzle geometry will have to be considered in future simulations.

\section{ACKNOWLEDGMENTS}

The second author is grateful to the Centre National d'Etudes Spatiales (CNES) for financial support. The authors also gratefully acknowledge the Institut du Développement et des Ressources en Informatique Scientifique (IDRIS - CNRS) for providing computing time and for its technical assistance. A part of the computations was also carried out on one of the supercomputers of the CEA (the French Atomic Energy Agency).

\section{REFERENCES}

1 Zaman, K.B.M.Q., "Effect of the initial condition on subsonic jet noise," AIAA Journal, Vol. 23, No. 9, 1985, pp. 1370-1373.

2 Tam, C.K.W., "Jet noise: since 1952," Theoret. Comput. Fluid Dynamics, Vol. 10, 1998, pp. 393-405.

3 Wang, M., Freund J.B, and Lele, S.K. "Computational prediction of flowgenerated sound," Annu. Rev. Fluid. Mech., Vol. 38, 2006, pp. 483-512.

4 Zhao, W., Frankel, S.H., and Mongeau, L., "Large eddy simulations of sound radiation from subsonic turbulent jets," AIAA Journal, Vol. 39, No. 8, 2001, pp. 1469-1477. 
5 Bogey, C., Bailly, C., and Juvé, D., "Noise investigation of a high subsonic, moderate Reynolds number jet using a compressible LES," Theoret. Comput. Fluid Dynamics, Vol. 16, No. 4, 2003, pp. 273-297.

6 Bogey, C. and Bailly, C., "Investigation of downstream and sideline subsonic jet noise using Large Eddy Simulations," Theoret. Comput. Fluid Dynamics, Vol. 20, No. 1, 2006, pp. 23-40.

7 Bogey, C. and Bailly, C., "An analysis of the correlations between the turbulent flow and the sound pressure field of subsonic jets," J. Fluid Mech., Vol. 583, 2007, pp. 71-97.

8 Bodony, D.J. and Lele, S.K., "On using large-eddy simulation for the prediction of noise from cold and heated turbulent jets," Phys. Fluids, Vol. 17, 085103, 2005, pp. 118.

9 Rembold, B. and Kleiser, L., "Noise prediction of a rectangular jet using LargeEddy Simulation," AIAA Journal, Vol. 42, No. 9, 2004, pp. 1823-1831.

10 Bogey, C. and Bailly, C., "Effects of inflow conditions and forcing on a Mach 0.9 jet and its radiated noise," AIAA Journal, Vol. 43, No. 5, 2005, pp. 1000-1007.

11 DeBonis, J.R. and Scott, J.N., "Large-Eddy Simulation of a turbulent compressible round jet," AIAA Journal, Vol. 40, No. 7, 2002, pp. 1346-1354.

12 Biancherin, A., Lupoglazoff, N., Vuillot, F., and Rahier, G., "Comprehensive 3D unsteady simulations of subsonic and supersonic hot jet flow-fields. Part 2: acoustic analysis," AIAA 2002-2600.

13 Andersson, N., Eriksson, L.-E., and Davidson, L., "Large-Eddy Simulation of subsonic turbulent jets and their radiated sound," AIAA Journal, Vol. 43, No. 9, 2005, pp. 1899-1912.

14 Shur, M.L., Spalart, P.R. and Strelets, M.K., "Noise prediction for increasingly complex jets. Part I: Methods and tests," International Journal of Aeroacoustics, Vol. 4, No. 3\&4, 2005, pp. 213-246.

$15 \mathrm{Wu}$, X., Tristanto, I.H., Page, G.J., and McGuirk, J.J., "Influence of nozzle modelling in LES of turbulent free jets," AIAA 2005-2883.

16 Shen, H. and Tam, C.K.W, "Three-Dimensional Numerical Simulation of the Jet Screech Phenomenon," AIAA Journal, Vol. 40, No. 1, 2002, pp. 33-41.

17 Al-Qadi, I.M.A. and Scott, J.N., "High-order three dimensional numerical simulation of a supersonic rectangular jet," AIAA 2003-3238.

$18 \mathrm{Li}$, X.D. and Gao, J.H., "Numerical simulation of the generation mechanism of axisymmetric supersonic jet screech tones," Phys. Fluids, Vol. 17, No. 8, 2005, 085105.

19 Berland, J., Bogey, C., and Bailly, C., "Numerical study of screech generation in a planar supersonic jet," Phys. Fluids, Vol. 19, 2007, 075105, pp. 1-14.

20 Viswanathan, K. and Clark, L.T., "Effect of nozzle internal contour on jet aeroacoustics," International Journal of Aeroacoustics, Vol. 3, No. 2, 2004, pp. $103-135$ 
21 Zaman, K.B.M.Q., "Far-field noise of a subsonic jet under controlled excitation," J. Fluid Mech., Vol. 152, 1985, pp. 83-111.

22 Hussain, A.K.M.F. and Zedan, M.F., "Effects of the initial condition on the axisymmetric free shear layer: Effects of the initial fluctuation level," Phys. Fluids, Vol. 21, No. 9, 1978, pp. 1475-1481.

23 Husain, Z.D. and Hussain, A.K.M.F., "Axisymmetric mixing layer: influence of the initial and boundary conditions," AIAA Journal, Vol. 17, No. 1, 1979, pp. 48-55.

24 Zaman, K.B.M.Q., "Flow field and near and far sound field of a subsonic jet," J. Sound Vib., Vol. 106, No. 1, 1986, pp. 1-16.

25 Crighton, D.G., "Acoustics as a branch of fluid mechanics," J. Fluid Mech., Vol. 106, 1981, pp. 261-298.

26 Bridges, J.E. and Hussain, A.K.M.F., "Roles of initial conditions and vortex pairing in jet noise," J. Sound Vib., Vol. 117, No. 2, 1987, pp. 289-311.

27 Viswanathan, K., “Aeroacoustics of hot jets," J. Fluid Mech., Vol. 516, 2004, pp. 39-82.

28 Bogey, C. and Bailly, C., "Computation of a high Reynolds number jet and its radiated noise using LES based on explicit filtering," Computers and Fluids, Vol. 35, No. 10, 2006, pp. 1344-1358.

29 Uzun, A. and Hussaini, M., "High frequency noise generation in the near-nozzle region of a jet," AIAA 2006-2499.

30 Bogey, C. and Bailly, C., "A family of low dispersive and low dissipative explicit schemes for flow and noise computations," J. Comput. Phys., Vol. 194, No. 1, 2004, pp. 194-214.

31 Berland, J., Bogey, C., Marsden O., and Bailly, C., "High-order, low dispersive and low dissipative schemes for multiple-scale and boundary problems," J. Comput. Phys., Vol. 224, 2007, pp. 637-662.

32 Constantinescu, G.S. and Lele, S.K., "A highly accurate technique for the treatment of flow equations at the polar axis in cylindrical coordinates using series expansions," J. Comput. Phys., Vol. 183, 2002, pp. 165-186.

33 Bogey, C. and Bailly, C., "Large Eddy Simulations of transitional round jets: influence of the Reynolds number on flow development and energy dissipation," Phys. Fluids, Vol. 18, No. 6, 2006, 065101, pp. 1-14.

34 Bogey, C. and Bailly, C., "Decrease of the effective Reynolds number with eddyviscosity subgrid-scale modeling," AIAA Journal, Vol. 43, No. 2, 2005, pp. 437-439.

35 Bogey, C. and Bailly, C., "Large Eddy Simulations of round free jets using explicit filtering with/without dynamic Smagorinsky model," Int. J. Heat and Fluid Flow, Vol. 27, No. 4, 2006, pp. 603-610.

36 Eggels, J.G.M., Unger, F., Weiss, M.H., Westerweel, J., Adrian, R.J., Friedrich, R., and Nieustadt, F.T.M., "Fully developed turbulent pipe flow: a comparison between direct numerical simulation and experiment," J. Fluid Mech., Vol. 268, 1994, pp. 175-209. 
37 Bogey, C., Barré, S., and Bailly, C., "Direct computation of the noise generated by a hot coaxial jet," AIAA 2007-3587.

38 Lau, J.C., Morris, P.J., and Fisher, M.J., "Measurements in subsonic and supersonic free jets using a laser velocimeter," J. Fluid Mech., Vol. 93, No. 1, 1979, pp. 1-27.

39 Arakeri, V.H., Krothapalli, A., Siddavaram, V., Alkislar, M.B., and Lourenco, L., "On the use of microjets to suppress turbulence in a Mach 0.9 axisymmetric jet," J. Fluid Mech., Vol. 490, 2003, pp. 75-98.

40 Raman, G., Rice, E.J., and Reshotko, E., "Mode spectra of natural disturbances in a circular jet and the effect of acoustic forcing," Exp. Fluids, Vol. 17, 1994, pp. 415-426.

41 Islam, M.T. and Ali, M.A.T, "Mean velocity and static pressure distribution of a circular jet," AIAA Journal, Vol. 35, No. 1, 1997, pp. 196-197.

42 Michalke, A., "Survey on jet instability theory," Prog. Aerospace Sci., Vol. 21, 1984, pp. 159-199.

43 Bogey, C., Barré, S., Fleury, V., Bailly, C., and Juvé, D., "Experimental study of the spectral properties of near-field and far-field jet noise," International Journal of Aeroacoustics, Vol. 6, No. 2, 2007, pp. 73-92.

44 Maestrello, L., "Two points correlations of sound pressure in the far field of a jet: Experiment," NASA-TMX-72835, 1976.

45 Gloerfelt, X., Bogey, C., and Bailly, C., "Numerical evidence of mode switching in the flow-induced oscillations by a cavity," International Journal of Aeroacoustics, Vol. 2, No. 2, 2003, pp. 193-217.

46 Jordan, P., Gervais, Y., Valière, J.-C., and Foulon, H., "Final results from single point measurements," Project deliverable D3.4, JEAN - EU 5th Framework Programme, G4RD-CT2000-00313, 2002.

47 Tanna, H.K., “An experimental study of jet noise. Part I: Turbulent mixing noise," J. Sound Vib., Vol. 50, No. 3, pp. 405-428. 